\title{
Factorial Structure of Some Biological and Physical Measurements for Handball Goalkeepers
}

Abdel Moneim Bedier Al Qusair*, Mohamed Ashraf Mohamed Kamel**, Mohamed Masoud Ibrahim Sharaf***

\begin{abstract}
Sports scientific researches and studies are concerned with determining specifications required by each type of different sports activities and conditions that must be met in players to fit in with the type of practitioner activity. Selection based on scientific grounds contribute sports achievement.

Handball require special features in each player depending on his play position, handball goalkeeper has special requirements which may not be required in other players, that is to enable him to carry out his duties in variety of situations exposed during the games.

Handball is one of sports activities, which reached level of maturity and progress as a result of experts' interest in studying and addressing various problems in scientific methods to upgrade their players and access highest levels.

Handball goalkeeper efficiency directly affects the team results. As far as his physical ability high, as well as biological systems' performance level; as far as team results affects; because of his ability to continuity in performance with same starting level throughout the game to end.

Handball goalkeeper - due to his distinctive role and effective role - must be distinguished from the rest of the team with some physical, and physical, psychological and physiological criteria as well as the ability to predict the level which can be achieved be him and possibility of continue playing handball with high efficiency as goalkeeper is most important position in the in the team.

This research aiming to determine factorial structure of some biological (morphological, and physiological) measurements for handball goalkeepers, study sample included (14) handball goalkeepers from nine first grade clubs as follows: "Ahli (2), Zamalek (1), Police Union (2), Nasr City (1), Armed forces (1), Damanhour games (2), Olympic (2), Sporting (2), Smouha (1)".

Results could determine five basic factors in morphological measurements (Abdomen depth, Skinfolds thickness for iliac prominence, Overall height), in physiological measurements (emotional stability) and in physical measurements( speed endurance.)

Most important recommendation were that there is necessity to focus on morphological, physiological and physical criteria extracted in this study (Speed endurance, Abdomen depth, Skinfolds thickness for iliac prominence, Overall height and Emotional stability) which could represent scientific base of handball goalkeepers selection, Giving attention to morphological, physiological and physical aspects as an important indicator for players' general physical condition, Selection process based on sound scientific principles, Choosing appropriate anticipated handball goalkeepers, Organizing and directing training programs for handball goalkeepers and upgrading handball sport at national level, developing handball sport at national level.
\end{abstract}

* Prof. Dr. Abdel Moneim Bedier Al Qusair Sports Physiology Professor, sport Biological and Health Sciences department, Faculty of Physical Education for Men, Alexandria University, Egypt.

** Asst. Prof. Dr. Mohamed Ashraf Kamel Assistant Professor, sports training department, Faculty of Physical Education for Men, Alexandria University, Egypt.

*** Dr./ Mohamed Masoud Ibrahim Sharaf Lecturer, sport Biological and Health Sciences department, Faculty of Physical Education for Men, Alexandria University, Egypt. 


\section{Introduction}

Q ports scientific researches and studies are concerned with determining specifications required by each type of different sports activities and conditions that must be met in players to fit in with the type of practitioner activity. Selection based on scientific grounds contribute sports achievement.

Recent world have remarkable development in basic life areas which affects athletic performance's level. This is evident by its improvements it year after year in international and world tournaments and Olympics. However with the witnessed tremendous progress in our contemporary world in sport achievement levels, discovering performance characteristics and requirements of various sports activities has great deal of importance; if we aiming are to achieve high and sophisticated standards for our Egyptian teams (Rasha EMira, 2009: p2)

Handball require special features in each player depending on his play position, handball goalkeeper has special requirements which may not be required in other players, that is to enable him to carry out his duties in variety of situations exposed during the games.

Handball is one of sports activities, which reached level of maturity and progress as a result of experts' interest in studying and addressing various problems in scientific methods to upgrade their players and access highest levels.

Handball goalkeeper efficiency directly affects the team results. As far as his physical ability high, as well as biological systems' performance level; as far as team results affects; because of his ability to continuity in performance with same starting level throughout the game to end. (Kamal Darwish et al(1998): p14)

To determine criteria for each sporting activity or players positions; we must get it from research applied on high levels athletes, and as more as research done on high level athlete as more as its results more conforming for necessary success criteria in sports activity (Ali El Bel,1996 :p 87).
To access high performance levels; Handball goalkeeper must have many Biological (morphological, physiological) and physical criteria which help him to perform his duties distinctly; For these specifications must be obtained through research results applied on high in all sports, as well as players positions.

Handball goalkeeper - due to his distinctive role and effective role - must be distinguished from the rest of the team with some physical, and physical, psychological and physiological criteria as well as the ability to predict the level which can be achieved be him and possibility of continue playing handball with high efficiency as goalkeeper is most important position in the in the team.

Many handball experts and specialists in the opinion that goalkeeper is the most important position in the team either in defense or attack, because he is the player who has been guard team goal which is the aim of opposing team. Goalkeeper modest or weak performance level is the key factor in his team loss, while his outstanding performance contributes positively to team results. Goalkeeper performance method is built on what he has from physical and skill capabilities. Players performance level rise with promoting and developing their physical traits (Kamal Darwish et al,1998 : 17) (Mohammed Lotfy Taha, 2002 : 113) (Monir Gerges, 2004: p19).

From this point of view handball goalkeeper should have distinctive Biological ( morphological, physiological ) and physical criteria to qualify him for excellence in skills performance, Hence study importance appear in terms of identifying most important Biological ( morphological, physiological ) measurements and physical guards handball goal.

\section{Research Objective}

This research aiming to determine factorial structure of some Biological and physical measurements for handball goalkeepers 


\section{Research Procedures}

\section{Research Methodology}

Descriptive approach was used with survey style because of its appropriateness of study nature.

Study sample: study sample included (14) handball goalkeepers from nine first grade clubs as follows: "Ahli (2), Zamalek (1), Police Union (2), Nasr City (1), Armed forces (1), Damanhour games (2), Olympic (2), Sporting (2), Smouha (1)" .

\section{Research Domains}

\section{Time domain:}

Study measurements was carried out during 2011/2012 season while goalkeepers for different national team gathering in Maadi Olympic Center in the period June 2nd to June 7 th.

Study measurements were carried out on handball goalkeepers in Alexandria during 2011/2012 season in the period July 9th to July 10th 2012.

\section{Spatial domain:}

Study measurements were applied in hall 2 in Maadi Olympic Center, Cairo

Study measurements were applied on handball goalkeepers in covered hall (Olympic - Sporting - Smouha - clubs and in Damanhour stadium in Behiara governorate province lake).

\section{Measurements used in the study:}

First: Biological measurements have been divided (morphological measurements physiological measurements)

\section{1. morphological measurements: -}

\section{- Total body height:}

Total body height measured using restameter (Mohamed Hasanin, 2003: p51).

\section{- Upper Limb height:}

Measurement is done from sitting position on a backless seat.

\section{- Lower Limb Height:}

Lowing Limb Height is measured using measuring tape from thigh femoral nick to land (Mohamed Hasanin, 2003: p35)

- Weight: weight estimated by using medical scale. (Mohamed Hasanin, 2003:p 56)

\section{- Circumference Measurement}

Measuring tape is used to determine different body parts circumference (neck - shoulders upper arm - chest - waist- thigh - knee - leg calf) (Mohamed Hasani, 2003: pp35, 54).

\section{- Width Measurement}

Belfometer is used to measure widths, it is a device similar to compass, where its twi ends fixed on measuring points and its scale indicate the width if measured area (Shoulder - iliac bone - hips) (Mohamed Hasanin, 2003:p 54, 55).

\section{- Depth Measurements}

Belfometer is used to measure depths ( (Mohamed Hasanin, 2003: p55)

\section{Body Mass Index ( BMI):}

BMI is calculated by dividing body weight in $\mathrm{kg}$ by the height square in meters, as follows:

Body mass index $(\mathrm{kg} \mathrm{2})=$ weight $(\mathrm{kg}) \div$ height square (meters) (Hazaa Ben Al Hazaa, 201: p103) (Miller,1995: p622) (Kamal darwish et al,1998:p 14) (Baumgartner,1995:p 292) (Davis et al,1995:p 587).

- Skin folds thickness measurements: skin fold caliper used to measure skin folds

\section{Measurement Places:}

Area behind humerus in triceps muscle area, it is vertical skin fold - Chest area, it is diagonal skin fold -sub scapula area, it is diagonal skin fold - abdominal area, It is vertical skin fold Sup iliac prominence, it is diagonal skin fold. Iliac prominence, it is diagonal skin fold.-Thigh area, it is vertical skin fold.-Medial side of calf leg, it is vertical skin fold (Abo EL Eal ABd ElFatah,1988 : pp333-335)

\section{- Palm area measurement:}

It is measured using measuring tape from the middle of the wrist to the end of the middle finger while extended (Mohamed Hasanin, 2004:p 52) 


\section{Physiological measurements:}

\section{- Measuring heart rate at rest and effort: -}

In rest: stopwatch used to measure the time of 30 pulses then pulse rate extracted (Abo ElEla Abd El Fatah,2004:pp58-59), (Blair V., 1999: p54)

In effort: subject do a particular effort such as a surprise attack several times, measurement is done immediately after the effort and after every two minutes till the heart returns to its rest rate (Zakia Fathy et al, 2001:p 233), (Foss M. et al, 1998: p53)

\section{Vital capacity measurement:}

Lungs vital capacity measured by dry Spiro meter o device, (Zakia Fathi et al, 2001:p4), (Mohamed Saad ElDin, 1997 :pp 257 - 258)

\section{- Measurement of Maximum Oxygen Uptake VO2max:}

Fox equation used, which is considered easy and simple equation to estimate maximum oxygen uptake, which created a linear relationship correlated with direct method sub-maximal heart recorded during the fifth minute on the with 150 watts intensity:

Maximum Oxygen Uptake (VO2MAX) $=6.3$ 0.093 X sub-maximal HR

Emotional stability measurement: - Hand stability device used to measure emotional stability (Mohammed Taha, 2004: pp113-114)

\section{Highest Motor frequency measurement :}

Paper and Pencil test used to measure highest motor frequency

(Moihammed Tahs, 2002: pp111-112)

\section{- Motor Coordination measurement:}

Rope jump is used to measure motor coordination. (Mohamed Hassanin, 2004 :pp 320-321).

Measuring reaction speed: ruler test is used to measure reaction speed. S (Mohamed Hassanin, 2004: pp275- 276)

\section{Neuromuscular coordination measurement}

Tennis ball throwing on wall used to measure neuromuscular coordination
5 Tennis ball, Wall, line drawn on land; five meters away from the wall. (Mohammed hassanin, 2004:p 328)

\section{Physical measurements:}

\section{- Accuracy Measurement:}

Shooting with hand on overlapping rectangle tests used to measure accuracy. (Mohamed Hassanin, 2004:pp358-359)

\section{Balance measurement}

Standing with foot (longitudinal) on a beam test used to measure balance

(Mohamed Hassanin, 2004: pp344- 345) (Ted, A. et al., 1999: p229) (Mohamed Hassanin,2000:p 425) (Abo ElEla Abd Elfattah,1988: pp308-309)

\section{- Measure muscular endurance:}

Arm bent test from diagonal lying position used to measure muscular endurance

Mohamed Hassanin, 2004:pp 236- 237) (Mohamed Hassanin, 1995:p 241) (Abo ElEla Abd Elfatah, 1988: pp113 - 115) (Ted A. et al: 231).

\section{- Speed endurance measurement:}

100 meter sprint test used to measure speed endurance

- Muscle strength: represented in right and left handgrip strength, Manometer device is used to measure grip strength (Baumgartner,1995: p165) (Ted A. et al,1999 : 209)

\section{- Flexibility:}

Trunk bent forward from standing test used to measure flexibility

(Mohamed hassanin, 2004Lp265 ) (Ted A. et al , 1999:p 229).

\section{- Agility:}

Multi-direction run test is used to measure agility (Mohammed Hassanin, 2004: 279)

- Speed: 15 seconds running in place test used to measure speed (Mohamed Hassanin, 2004: p292)

- Ability: 
Long jump test is used to measure capacity (Mohamed Hassanin, 2004:p 307) (Ted A, 1999: p227) (Baumgartner et al, 1995: p230)

- Specific Power: To measure Specific Power Test was selected vertical jump of persistence (Sargent), (Mohamed Sobhy hassanin,1995: pp90-91)

\section{Administrative Procedures:-}

Before conducting research pilot and main studies, there were some procedures had to be taken to facilitate implementation of pilot and main studies to achieve study objectives, the following actions have been taken:

- Management Communications: approval obtained for conducting measurements on handball goalkeepers from from clubs, national teams goalkeepers' coach, Manager Of Maadi Olymic center

- Preparing measurements registration forms: registration form have been prepared for each measurement type (Morphology measurements form - physical measurements Form physiological measurements Communications)

1. Preparation of place, tools and devices: Morphological, physical and some physiological measurements done in Hall (2) Maadi Olympic Center, some physiological measurements were conducted in International Cairo Stadium Medical Center, before conducting tests validity of devices, tools and forms used in research has been conformed taking into account the technical requirements to ensure access to accurate results, free from the errors that result from inappropriate devices and instruments.

\section{Main study}

Research morphological, physiological and physical measurements were conducted on first class goalkeepers in covered hall No. 2, Maadi Olympic Center in Cairo during the period of June 6th to June 7th. Also measurements were conducted on Alexandria handball goalkeepers through tests which got overall experts approval in the period from July 9th To July 10th 2012.

- Statistical Work: (arithmetic mean, standard deviation, skewness, kurtosis, factor analysis) 


\section{Results and Discussion}

First: Factor analysis of biological measurements:

\section{Factorial analysis of morphological variables}

Table (1)

Mean, standard deviation, skewness coefficient, and kurtosis of research sample in morphological measurements

\begin{tabular}{|c|c|c|c|c|c|c|}
\hline \multicolumn{2}{|l|}{ Variables } & $\begin{array}{c}\text { Measurement } \\
\text { unit }\end{array}$ & Mean & $\begin{array}{c}\text { Standard } \\
\text { Deviation }\end{array}$ & $\begin{array}{c}\text { Skewness } \\
\text { factor }\end{array}$ & Kurtosis \\
\hline \multicolumn{2}{|c|}{ Body weight } & $\mathrm{Kg}$ & 83.71 & 6.94 & -.96 & .46 \\
\hline \multirow[t]{3}{*}{ Heights } & Overall height & $\mathrm{Cm}$ & 179.57 & 7.47 & -.52 & -.27 \\
\hline & Lower limb & $\mathrm{Cm}$ & 91.43 & 4.82 & .29 & -.76 \\
\hline & Upper limb & $\mathrm{Cm}$ & 88.14 & 5.79 & -.80 & 1.13 \\
\hline \multirow[t]{8}{*}{ Circumferences } & Neck circumference & $\mathrm{Cm}$ & 37.79 & 6.74 & -.28 & .26 \\
\hline & Shoulders circumference & $\mathrm{Cm}$ & 110.43 & 12.74 & -.13 & -.78 \\
\hline & Humerus circumference & $\mathrm{Cm}$ & 33.43 & 6.87 & .36 & -.68 \\
\hline & Chest circumference & $\mathrm{Cm}$ & 98.21 & 12.86 & -.04 & -.87 \\
\hline & Waist circumference & $\mathrm{Cm}$ & 88.86 & 8.83 & -1.03 & -.09 \\
\hline & Thigh circumference & $\mathrm{Cm}$ & 59.71 & 9.23 & .06 & -1.10 \\
\hline & Knee circumference & $\mathrm{Cm}$ & 41.79 & 3.93 & 1.26 & .77 \\
\hline & Calf circumference & $\mathrm{Cm}$ & 41.57 & 5.50 & .72 & -.15 \\
\hline \multirow[t]{3}{*}{ Widths } & Shoulder width & $\mathrm{Cm}$ & 15.07 & 1.75 & -.45 & -1.18 \\
\hline & Iliac bone width & $\mathrm{Cm}$ & 13.82 & 1.28 & -.79 & .300 \\
\hline & Hips width & $\mathrm{Cm}$ & 6.86 & 1.50 & -.02 & -.11 \\
\hline \multirow[t]{3}{*}{ Depths } & Chest depth & $\mathrm{Cm}$ & 7.50 & 1.34 & -.89 & -.14 \\
\hline & Abdomen depth & $\mathrm{Cm}$ & 8.39 & 1.43 & .25 & -.52 \\
\hline & Pelvic depth & $\mathrm{Cm}$ & 7.61 & 1.48 & .29 & .310 \\
\hline \multicolumn{2}{|c|}{ Body Mass index (BMI) } & $\%$ & 25.82 & 2.52 & .29 & 0.40 \\
\hline \multirow[t]{8}{*}{ Skinfolds thicknesses } & Behind humerus & millimeter & 11.07 & 3.08 & -.13 & -1.61 \\
\hline & Chest & millimeter & 14.36 & 6.32 & .36 & -0.92 \\
\hline & sub scapula area & millimeter & 12.21 & 5.18 & .52 & -1.46 \\
\hline & Sup iliac prominence & millimeter & 15.71 & 5.54 & .38 & -0.96 \\
\hline & iliac prominence & millimeter & 16.21 & 6.70 & .42 & -0.34 \\
\hline & Abdominal area & millimeter & 20.50 & 8.14 & .24 & -1.52 \\
\hline & Thigh area & millimeter & 16.71 & 6.78 & .38 & -0.88 \\
\hline & Medial side of calf & millimeter & 5.57 & 2.17 & 0.44 & -1.42 \\
\hline \multicolumn{2}{|c|}{ Palm length } & $\mathrm{Cm}$ & 23.29 & 1.90 & -0.11 & -1.06 \\
\hline
\end{tabular}

Table No. (1) results revealed that $(-1.61,1.13)$ i.e. in between \pm 3 and this gives an morphological variable for research sample direct indication that sample represents normal members follow normal frequency distribution society and free of abnormal distribution effects. (normal curve) where skew factors were between 
Table (2)

Correlation matrix between morphological indicators for research sample

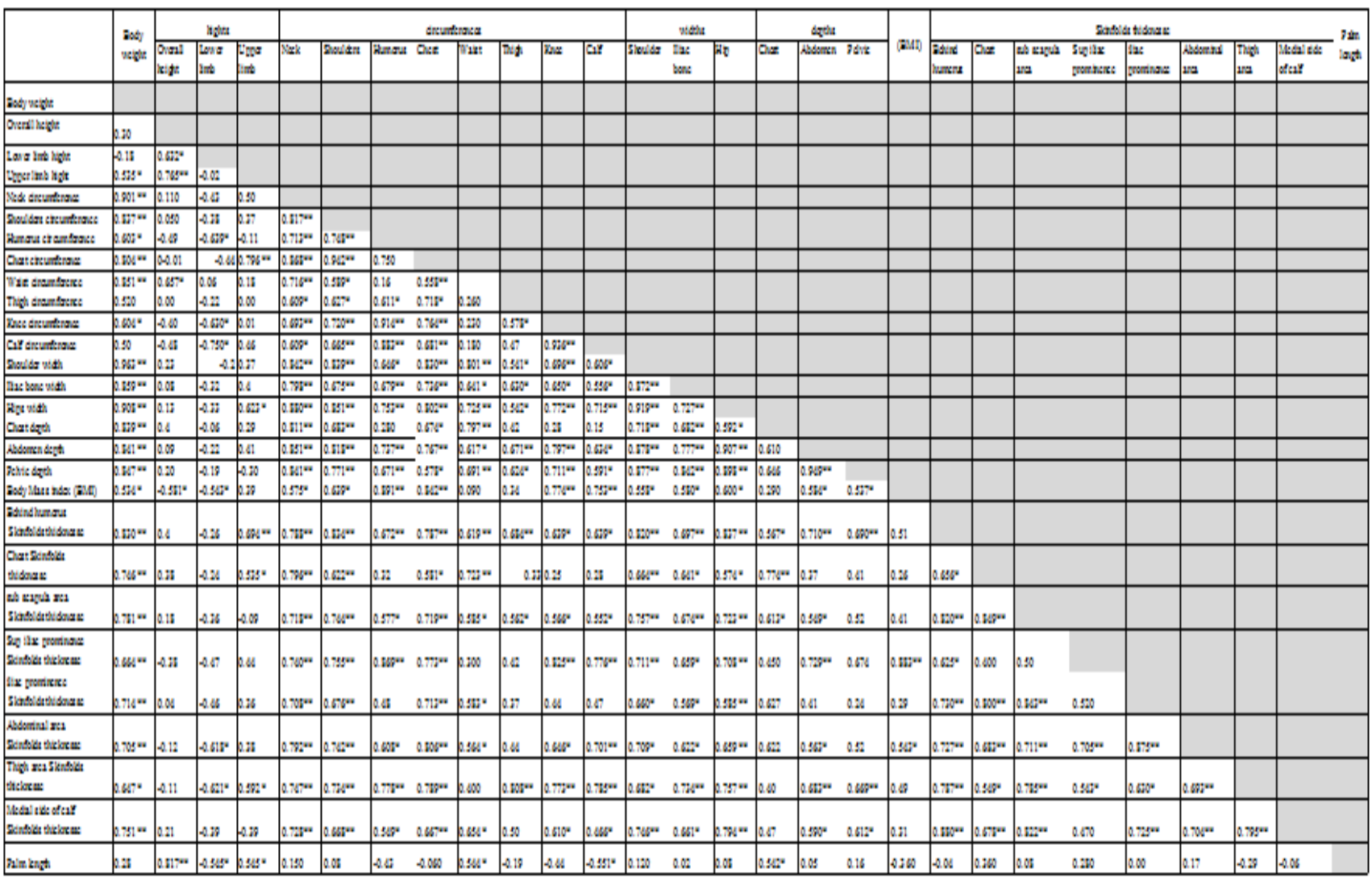


Table (3)

Rotated Component Matrix for goalkeepers morphological variables

Final results after removing loads less than $\pm(0.35)$

Extraction Method: Principal Component Analysis.

Rotation Method: Varimax with Kaiser Normalization

\begin{tabular}{|c|c|c|c|c|c|}
\hline \multirow{2}{*}{ Morphological Variables } & \multicolumn{4}{|c|}{ Factor } & \multirow{2}{*}{ Communalities } \\
\hline & First & Second & Third & Fourth & \\
\hline Abdomen depth & 0.936 & & & & 0.963 \\
\hline Pelvic depth & 0.918 & & & & 0.95 \\
\hline Shoulder width & 0.817 & 0.466 & & & 0.924 \\
\hline Hips width & 0.817 & 0.398 & & & 0.907 \\
\hline Body weight & 0.79 & 0.556 & & & 0.969 \\
\hline Skinfolds thickness, sup iliac prominence & 0.774 & & -0.487 & & 0.932 \\
\hline Neck circumference & 0.76 & 0.565 & & & 0.918 \\
\hline Shoulders circumference & 0.754 & 0.494 & & & 0.851 \\
\hline Iliac bone width & 0.741 & 0.401 & & & 0.765 \\
\hline Chest circumference & 0.726 & 0.487 & & & 0.856 \\
\hline Humerus circumference & 0.707 & & -0.605 & & 0.955 \\
\hline Knee circumference & 0.702 & & -0.543 & & 0.922 \\
\hline Body Mass index (BMI) & 0.687 & & -0.629 & & 0.929 \\
\hline Skinfolds thickness, iliac prominence & 0.601 & 0.54 & & 0.413 & 0.827 \\
\hline Skinfolds thickness, iliac prominence & & 0.901 & & & 0.909 \\
\hline Skinfolds thickness, iliac prominence & & 0.872 & & & 0.89 \\
\hline Skinfolds thickness, Abdominal area & 0.425 & 0.77 & & & 0.881 \\
\hline Skinfolds thickness, sub scapula area & 0.394 & 0.757 & & & 0.851 \\
\hline Skinfolds thickness, Medial side of calf & 0.396 & 0.648 & & 0.535 & 0.863 \\
\hline Upper limb height & & 0.621 & 0.616 & & 0.876 \\
\hline Chest depth & 0.594 & 0.606 & 0.365 & & 0.873 \\
\hline Waist circumference & 0.532 & 0.577 & 0.531 & & 0.905 \\
\hline Overall height & & & 0.96 & & 0.981 \\
\hline Palm length & & & 0.866 & & 0.886 \\
\hline Lower limb & & -0.439 & 0.748 & & 0.786 \\
\hline Calf circumference & 0.527 & & -0.653 & & 0.904 \\
\hline Skinfolds thickness, thigh & 0.48 & 0.465 & & 0.651 & 0.961 \\
\hline Thigh circumference & 0.537 & & & 0.635 & 0.718 \\
\hline Eigen value & 10.44 & 7 & 5.1 & 2.41 & 24.95 \\
\hline Variance percentage after rotation & $37.27 \%$ & $25.01 \%$ & $18.22 \%$ & $8.62 \%$ & \\
\hline
\end{tabular}

Table (3) results reveal that factorial analysis due to the fact that it has less than three after rotation of morphological indicators variables, variance for first factor was $37.27 \%$, matrix for handball goalkeepers resulted in four second factor $25.01 \%$, third factor $18.22 \%$ and factors, the fourth factor have been excluded , fourth factor was $8.62 \%$

Table (4)

Final extracted factors from factorial analysis using orthogonal rotation of morphological variables for handball goalkeepers

\begin{tabular}{|c|c|c|}
\hline Factor Rank & Indicator Name & Loading on Factor \\
\hline First & Abdomen depth & 0.936 \\
\hline Second & Skinfolds thickness, iliac prominence & 0.901 \\
\hline Third & Overall height & 0.960 \\
\hline
\end{tabular}

Table (4) results reveal that factorial analysis of Skinfolds thickness of iliac prominence came in morphological indicators for goalkeepers second factor worth loading of 0.901 and resulted in three key factors; where abdomen overall height came in third factor with loading depth came in first factor with loading of 0.948, of 0.960 


\section{Factorial analysis of physiological variables}

Table (5)

Mean, standard deviation, skewness coefficient, and kurtosis of research sample in physiological measurements

\begin{tabular}{|c|c|c|c|c|c|c|}
\hline \multicolumn{2}{|c|}{ Statistical indicators } & $\begin{array}{c}\text { Measurement } \\
\text { unit }\end{array}$ & Mean & $\begin{array}{c}\text { Standard } \\
\text { Deviation }\end{array}$ & $\begin{array}{l}\text { Skewness } \\
\text { factor }\end{array}$ & Kurtosis \\
\hline \multirow{2}{*}{ Heart rate } & Rest & Pulse/minute & 60.71 & 7.76 & 0.03 & -1.1 \\
\hline & Effort & Pulse/minute & 170.93 & 21.46 & -0.33 & -1.4 \\
\hline \multicolumn{2}{|c|}{ Vital capacity } & Milliliter/minute & 4155.36 & 4155.36 & 873.21 & -0.26 \\
\hline \multicolumn{2}{|c|}{$\begin{array}{l}\text { Absolute Maximum Oxygen Uptake (VO2max } \\
\text { absolute) }\end{array}$} & Milliliter/minute & 3232.14 & 3232.14 & 728.32 & -0.11 \\
\hline \multicolumn{2}{|c|}{$\begin{array}{c}\text { Relative Maximum Oxygen Uptake (VO2max } \\
\text { relative) }\end{array}$} & Milliliter/minute/Kg. & 38.07 & 38.07 & 8.07 & -0.11 \\
\hline \multicolumn{2}{|c|}{ Reaction speed } & Second & 12.14 & 12.14 & 1.75 & -0.15 \\
\hline \multicolumn{2}{|c|}{ Neuromuscular coordination } & Second & 14.21 & 14.21 & 4.92 & -0.24 \\
\hline \multicolumn{2}{|c|}{ Emotional stability } & Number & 3.50 & 3.5 & 1.51 & -0.36 \\
\hline \multicolumn{2}{|c|}{ Motor coordination } & Second & 3.38 & 3.38 & 1.5 & -0.44 \\
\hline \multirow{4}{*}{ Highest Motor frequency } & 1st attempt & Number & 58.5 & 12.77 & 0.1 & -1.07 \\
\hline & 2nd attempt & Number & 52.93 & 10.91 & 0.29 & -1.21 \\
\hline & 3rd attempt & Number & 53.21 & 11.06 & -0.23 & -1.64 \\
\hline & 4th attempt & Number & 52.57 & 11.82 & 0.22 & -0.83 \\
\hline
\end{tabular}

Table No. (5) results revealed that physiological 0.64 ) i.e. in between \pm 3 and this gives an direct variable for research sample members follow indication that sample represents normal society normal frequency distribution (normal curve) and free of abnormal distribution effects.

where skew factors were between $(-1.80$ to - 


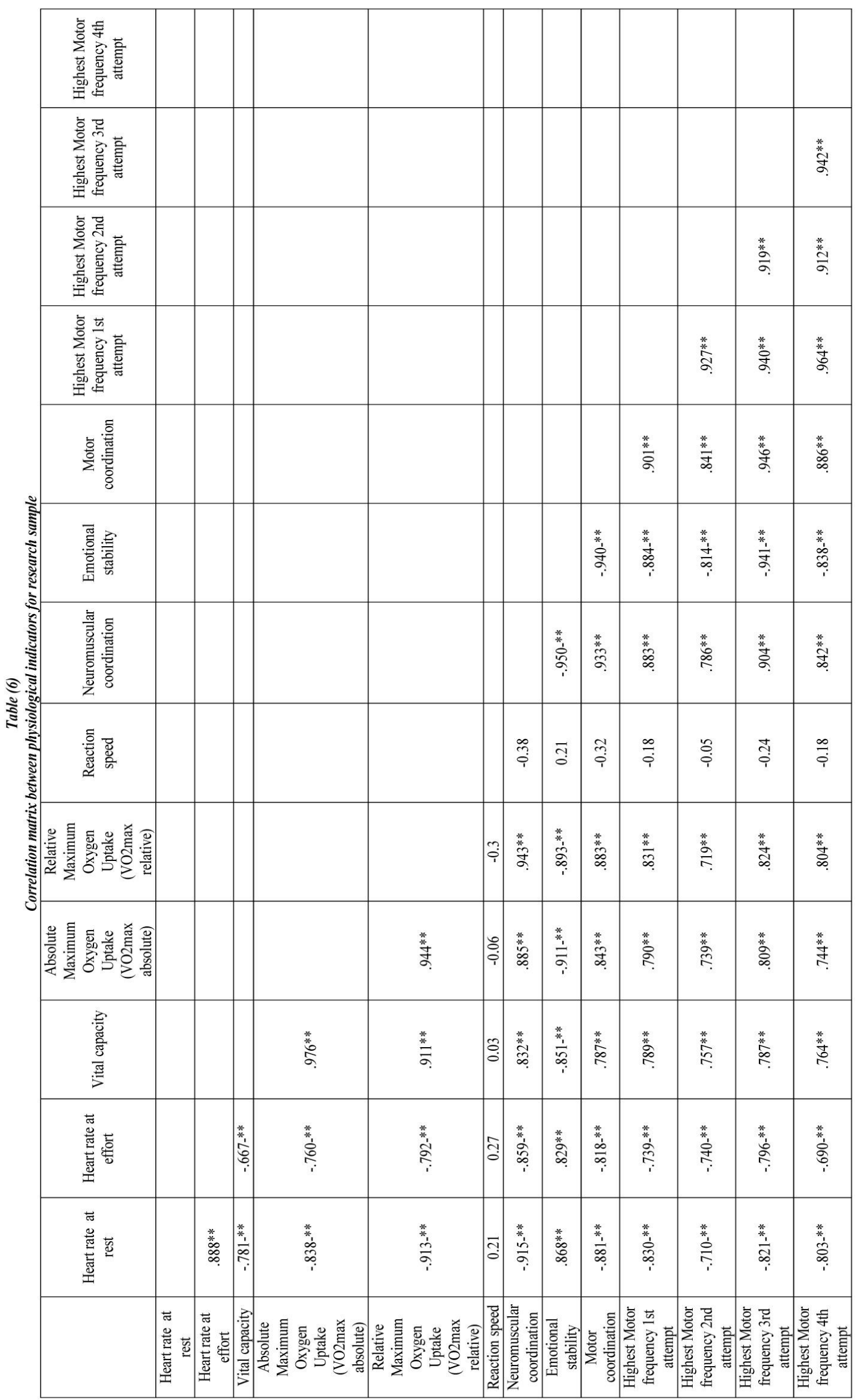


Table (7)

Rotated Component Matrix for goalkeepers morphological variables

Final results after removing loads less than $\pm(0.35)$

Extraction Method: Principal Component Analysis.

Rotation Method: Varimax with Kaiser Normalization

\begin{tabular}{|c|c|c|c|}
\hline \multirow{2}{*}{ Physiological Variables } & \multicolumn{2}{|c|}{ Factor } & \multirow{2}{*}{ Communalities } \\
\cline { 2 - 3 } & First & Second & \\
\hline Emotional stability & -0.953 & & 0.93 \\
\hline Highest Motor frequency 3rd attempt & 0.942 & & 0.915 \\
\hline Highest Motor frequency 1st attempt & 0.939 & & 0.89 \\
\hline Absolute Maximum Oxygen Uptake (VO2max absolute) & 0.935 & & 0.875 \\
\hline Motor coordination & 0.932 & & 0.935 \\
\hline Neuromuscular coordination & 0.93 & & 0.966 \\
\hline Vital capacity & 0.921 & & 0.872 \\
\hline Relative Maximum Oxygen Uptake (VO2max relative) & 0.92 & & 0.892 \\
\hline Highest Motor frequency 4th attempt & 0.912 & & 0.841 \\
\hline Heart rate at rest & -0.902 & & 0.852 \\
\hline Highest Motor frequency 2nd attempt & 0.901 & & 0.813 \\
\hline Heart rate at effort & -0.829 & & 0.76 \\
\hline Reaction speed & & 0.98 & 0.967 \\
\hline Eigen value & 10.13 & 1.38 & 11.51 \\
\hline Variance percentage after rotation & $77.93 \%$ & $10.59 \%$ & \\
\hline
\end{tabular}

Table (7) results reveal that factorial analysis the fact that it has less than three variables, after rotation of physiological indicators matrix variance for first factor was $77.93 \%$ and second for handball goalkeepers resulted in two factors, factor $10.59 \%$, third factor $18.22 \%$ and fourth the second factor have been excluded, due to factor was $8.62 \%$

Table (8)

Final extracted factors from factorial analysis using orthogonal rotation of physiological variables for handball goalkeepers

\begin{tabular}{|c|c|c|}
\hline Factor Rank & Indicator Name & Loading on Factor \\
\hline First & Emotional stability & -0.953 \\
\hline
\end{tabular}

Table (8) results reveal that factorial analysis of in one key factor; where emotional stability physiological indicators for goalkeepers resulted came in first factor with loading of 0.953 ,

Second: Factor analysis of the physical variables:

Table (9)

Mean, standard deviation, skewness coefficient, and kurtosis of research sample in physical measurements

\begin{tabular}{|c|c|c|c|c|c|}
\hline Sariables & $\begin{array}{c}\text { Measurement } \\
\text { unit }\end{array}$ & Mean & $\begin{array}{c}\text { Standard } \\
\text { Deviation }\end{array}$ & $\begin{array}{c}\text { Skewness } \\
\text { factor }\end{array}$ & Kurtosis \\
\hline Right Handgrip strength & Kg. & 47.21 & 7.45 & -0.85 & -0.42 \\
\hline Left Handgrip strength & Kg. & 42.5 & 8.7 & -0.43 & -1.12 \\
\hline Flexibility & Centimeter & 17.32 & 2.58 & 0.41 & 0.64 \\
\hline Speed & Number & 31.93 & 5.93 & 0.09 & -0.87 \\
\hline Agility & Second & 16.62 & 3.74 & 0.61 & -1.03 \\
\hline Capacity & Centimeter & 216.36 & 11.71 & -0.09 & -1.18 \\
\hline Power & Centimeter & 75.79 & 32.18 & -0.18 & -1.93 \\
\hline Accuracy & Marks & 10.21 & 3.36 & -0.6 & -0.57 \\
\hline Balance & Second & 11.57 & 3.73 & 1.14 & 2.17 \\
\hline Muscular endurance & Number & 42.36 & 7.88 & -0.09 & 0.16 \\
\hline Speed Endurance & Second & 13.25 & 2.04 & 0.71 & -0.6 \\
\hline
\end{tabular}

Table No. (9) results revealed that physical 2.17) i.e. in between \pm 3 and this gives an direct variable for research sample members follow indication that sample represents normal society normal frequency distribution (normal curve) and free of abnormal distribution effects. where skew factors were between $(-1.93$ to 
Table (10)

Correlation matrix between physical indicators for research sample

\begin{tabular}{|c|c|c|c|c|c|c|c|c|c|c|c|}
\hline & $\begin{array}{l}\text { Right } \\
\text { Handgr } \\
\text { ip } \\
\text { strengt } \\
\text { h }\end{array}$ & $\begin{array}{l}\text { Left } \\
\text { and } \\
\text { grip } \\
\text { streng } \\
\text { th }\end{array}$ & $\begin{array}{l}\text { Flexibil } \\
\text { ity }\end{array}$ & Speed & $\begin{array}{l}\text { Agilit } \\
\mathrm{y}\end{array}$ & $\begin{array}{l}\text { Capaci } \\
\text { ty }\end{array}$ & Power & $\begin{array}{l}\text { Accura } \\
\text { cy }\end{array}$ & $\begin{array}{l}\text { Balan } \\
\text { ce }\end{array}$ & $\begin{array}{l}\text { Muscul } \\
\text { ar } \\
\text { enduran } \\
\text { ce }\end{array}$ & $\begin{array}{l}\text { Speed } \\
\text { nduran } \\
\text { ce }\end{array}$ \\
\hline $\begin{array}{c}\text { Right } \\
\text { Handgri } \\
\mathrm{p} \\
\text { strength }\end{array}$ & & & & & & & & & & & \\
\hline $\begin{array}{c}\text { Left } \\
\text { Handgri } \\
\mathrm{p} \\
\text { strength }\end{array}$ & $.869 * *$ & & & & & & & & & & \\
\hline $\begin{array}{c}\text { Flexibili } \\
\text { ty }\end{array}$ & -0.44 & -0.47 & & & & & & & & & \\
\hline Speed & -0.15 & -0.13 & $0.566^{*}$ & & & & & & & & \\
\hline Agility & 0.15 & 0.17 & -.614-* & $\begin{array}{c}-.836- \\
* *\end{array}$ & & & & & & & \\
\hline $\begin{array}{c}\text { Capacit } \\
\text { y }\end{array}$ & -0.25 & -0.37 & $0.572 *$ & $\begin{array}{c}0.921^{*} \\
*\end{array}$ & $\begin{array}{c}-.816- \\
* *\end{array}$ & & & & & & \\
\hline Power & -0.17 & -0.19 & $0.589 *$ & $\begin{array}{c}0.853 * \\
*\end{array}$ & $\begin{array}{c}0940- \\
* *\end{array}$ & $\begin{array}{c}0.822 * \\
*\end{array}$ & & & & & \\
\hline $\begin{array}{c}\text { Accurac } \\
\mathrm{y}\end{array}$ & -0.12 & -0.01 & $0.605^{*}$ & $\begin{array}{c}0.782 * \\
*\end{array}$ & $\begin{array}{c}0779- \\
* *\end{array}$ & $0.624^{*}$ & $\begin{array}{c}0.780 * \\
*\end{array}$ & & & & \\
\hline Balance & -0.18 & -0.29 & 0.49 & $0.579 *$ & -0.48 & $0.632 *$ & 0.43 & 0.47 & & & \\
\hline $\begin{array}{c}\text { Muscula } \\
r \\
\text { enduran } \\
\text { ce }\end{array}$ & -0.05 & 0.1 & 0.52 & $\begin{array}{c}0.867 * \\
*\end{array}$ & $\begin{array}{c}-.787- \\
* *\end{array}$ & $\begin{array}{c}0.683 * \\
*\end{array}$ & $\begin{array}{c}0.713 * \\
*\end{array}$ & $\begin{array}{c}0.881 * \\
*\end{array}$ & $\begin{array}{c}0.600 \\
*\end{array}$ & & \\
\hline $\begin{array}{c}\text { Speed } \\
\text { Enduran } \\
\text { ce }\end{array}$ & 0.24 & 0.25 & -.611-* & $\begin{array}{c}0.880- \\
* *\end{array}$ & $\begin{array}{c}0.906^{*} \\
*\end{array}$ & $\begin{array}{c}0.842- \\
* *\end{array}$ & $\begin{array}{c}0.936- \\
* *\end{array}$ & $\begin{array}{c}0.887- \\
* *\end{array}$ & $\begin{array}{c}0.560- \\
*\end{array}$ & $\begin{array}{c}0.812- \\
* *\end{array}$ & \\
\hline
\end{tabular}

Table (11)

Rotated Component Matrix for goalkeepers physical variables

Final results after removing loads less than $\pm(0.35)$

Extraction Method: Principal Component Analysis.

Rotation Method: Varimax with Kaiser Normalization

\begin{tabular}{|c|c|c|c|}
\hline \multirow{2}{*}{ Physiological Variables } & \multicolumn{2}{|c|}{ Factor } & \multirow{2}{*}{ Communalities } \\
\cline { 2 - 3 } & First & Second & \\
\hline Speed Endurance & -0.948 & & 0.93 \\
\hline Speed & 0.943 & & 0.896 \\
\hline Agility & -0.924 & & 0.866 \\
\hline Muscular endurance & 0.918 & & 0.854 \\
\hline Power & 0.911 & & 0.846 \\
\hline Capacity & 0.897 & & 0.805 \\
\hline Accuracy & 0.854 & & 0.812 \\
\hline Flexibility & 0.619 & -0.507 & 0.64 \\
\hline Balance & 0.613 & & 0.444 \\
\hline Right Handgrip strength & & 0.979 & 0.962 \\
\hline Left Handgrip strength & & 0.924 & 0.858 \\
\hline Eigen value & 6.61 & 2.3 & 8.91 \\
\hline Variance percentage after rotation & $60.10 \%$ & $20.91 \%$ & \\
\hline
\end{tabular}

Table (11) results reveal that factorial analysis fact that it has less than three variables, variance after rotation of physical indicators matrix for for first factor was $60.10 \%$ and second factor handball goalkeepers resulted in two factors, the $10.59 \%$, third factor $18.22 \%$ and fourth factor second factor have been excluded, due to the was $20.91 \%$ 
Table (12)

Final extracted factors from factorial analysis using orthogonal rotation of physical variables for handball goalkeepers

\begin{tabular}{|c|c|c|}
\hline Factor Rank & Indicator Name & Loading on Factor \\
\hline First & Speed Endurance & -0.948 \\
\hline
\end{tabular}

Table (12) results reveal that factorial analysis endurance came in first factor with loading of of physiological indicators for goalkeepers 0.948, resulted in one key factor; where speed

Table (13)

Standard T-scores for morphological, physiological and physical variables derived from factor analysis of Handball goalkeepers

\begin{tabular}{|c|c|c|c|c|c|c|c|c|c|}
\hline \multicolumn{2}{|c|}{ Speed endurance } & \multicolumn{2}{c|}{ Abdomen depth } & \multicolumn{2}{c|}{$\begin{array}{c}\text { Skinfolds thickness, } \\
\text { iliac prominence }\end{array}$} & \multicolumn{2}{c|}{ Overall height } & \multicolumn{2}{c|}{ Emotional stability } \\
\hline $\begin{array}{c}\text { Raw } \\
\text { score }\end{array}$ & $\begin{array}{c}\text { Standard } \\
\text { score }\end{array}$ & $\begin{array}{c}\text { Raw } \\
\text { score }\end{array}$ & $\begin{array}{c}\text { Standard } \\
\text { score }\end{array}$ & $\begin{array}{c}\text { Raw } \\
\text { score }\end{array}$ & $\begin{array}{c}\text { Standard } \\
\text { score }\end{array}$ & $\begin{array}{c}\text { Raw } \\
\text { score }\end{array}$ & $\begin{array}{c}\text { Standard } \\
\text { score }\end{array}$ & $\begin{array}{c}\text { Raw } \\
\text { score }\end{array}$ & $\begin{array}{c}\text { Standard } \\
\text { score }\end{array}$ \\
\hline 11 & 70.36 & 6 & 68.23 & 7 & 69.07 & 165 & 30.49 & 0 & 37.9 \\
\hline 11.3 & 63.5 & 7 & 64.73 & 8 & 66.09 & 168 & 34.51 & 1 & 42.74 \\
\hline 11.5 & 61.05 & 8 & 57.74 & 12 & 55.65 & 172 & 39.86 & 2 & 47.58 \\
\hline 11.8 & 56.14 & 9 & 54.25 & 13 & 52.66 & 177 & 46.56 & 3 & 52.42 \\
\hline 12 & 53.69 & 9.5 & 47.25 & 17 & 51.17 & 178 & 47.9 & 4 & 57.26 \\
\hline 13.37 & 50.6 & 10.5 & 40.26 & 18 & 45.21 & 179 & 49.24 & 5 & 62.1 \\
\hline 14 & 43.88 & 11 & 33.27 & 20 & 43.71 & 180 & 50.57 & & \\
\hline 14.5 & 42.9 & & & 27 & 37.75 & 185 & 57.27 & & \\
\hline 15.5 & 41.43 & & & 29 & 36.26 & 187 & 59.94 & & \\
\hline 16 & 40.45 & & & & & & 189 & 62.62 & \\
\hline 17.4 & 38.98 & & & & & & & & \\
\hline
\end{tabular}

Table (13) demonstrates standard T-scores of morphological, physiological and physical variables derived from factor analysis of handball goalkeepers. For speed endurance lowest raw score were 17.4 which correspond to 38.98 standard score and the highest raw score were 11 which correspond to 70.36 noting that the arithmetic mean 13.37 correspond to 50.6 standard score. For abdomen depth lowest raw score were 11 which correspond to 33.27 standard score and the highest raw score were 6 which correspond to 68.23 noting that the arithmetic mean 9. correspond to 54.25 standard score. For skinfolds thickness of iliac prominence lowest raw score were 29 which correspond to 36.26 standard score and the highest raw score were 7 which correspond to 69.07 noting that the arithmetic mean 17 correspond to 51.7 standard score. For overall height lowest raw score were 165 which correspond to 30.49 standard score and the highest raw score were 190 which correspond to 63.96 noting that the arithmetic mean 179 correspond to 49.24 standard score. For emotional stability lowest raw score were 0 which correspond to 37.90 standard score and the highest raw score were 5 which correspond to 62.10 noting that the arithmetic mean 3 correspond to 52.42 standard score. 
Table (14)

Percentile ranking of morphological, physiological and physical variables derived from factor analysis of Handball goalkeepers

\begin{tabular}{|c|c|c|c|c|c|}
\hline $\begin{array}{c}\text { Percentile } \\
\text { rank }\end{array}$ & $\begin{array}{c}\text { Speed } \\
\text { endurance }\end{array}$ & $\begin{array}{c}\text { Abdomen } \\
\text { depth }\end{array}$ & $\begin{array}{c}\text { Skin folds thickness, } \\
\text { iliac prominence }\end{array}$ & Overall height & $\begin{array}{c}\text { Emotional } \\
\text { stability }\end{array}$ \\
\hline 95 & 11 & 6 & 7 & 190 & 0 \\
\hline 90 & 11.15 & 6.5 & 7.5 & 189.5 & 0 \\
\hline 85 & 11.3 & 7 & 11 & 188.5 & 0 \\
\hline 80 & 11.3 & 7 & 8 & 187 & 0 \\
\hline 75 & 11.45 & 7 & 8 & 185.5 & 0 \\
\hline 70 & 11.65 & 7.5 & 12 & 185 & 2.5 \\
\hline 65 & 11.8 & 8 & 17.5 & 183.75 & 1.25 \\
\hline 60 & 11.8 & 8 & 16 & 180 & 2 \\
\hline 55 & 11.95 & 8 & 12.25 & 179.5 & 2.5 \\
\hline 50 & 12.69 & 8 & 13 & 179 & 3 \\
\hline 45 & 13.53 & 8.25 & 18 & 178.25 & 3.75 \\
\hline 40 & 14 & 9 & 18 & 179 & 3 \\
\hline 35 & 14 & 9 & 18 & 177.5 & 4.5 \\
\hline 30 & 14.25 & 9.25 & 19 & 175.75 & 5 \\
\hline 25 & 14.75 & 9.5 & 20 & 169 & 5 \\
\hline 20 & 15.5 & 9.5 & 20 & 172 & 5 \\
\hline 15 & 15.88 & 10.25 & 25.25 & 166.5 & 5 \\
\hline 10 & 16.7 & 10.75 & 28 & & 5 \\
\hline 5 & 17.4 & 11 & 29 & & \\
\hline
\end{tabular}


Table (14) shows that for speed endurance percentile rank (5) correspond to 17.40 score, percentile rank (95) correspond to 12.69 and percentile rank (50) correspond to 12.69. For abdomen depth percentile rank (5) correspond to 11 score, percentile rank (95) correspond to 6 and percentile rank (50) correspond to 8. For skinfolds thickness for iliac prominence percentile rank (5) correspond to 29 score, percentile rank (95) correspond to 7 and percentile rank (50) correspond to 13. For overall height percentile rank (5) correspond to 165 score, percentile rank (95) correspond to 190 and percentile rank (50) correspond to 179.5. For emotional stability percentile rank (5) correspond to 5 score, percentile rank (95) correspond to 0 and percentile rank (50) correspond to 2.5

\section{Discussion of Factorial analysis results}

After arrived to inter-correlation matrix, factorial analysis aiming to interpret those correlation between measurements in the light of minimum number of factors, where are these factors descriptive names for series of tests or variables which are highly correlated, also it is supposed that they have common characteristics.

Hotelling's Principle Components method used in analyzing factorial matrix for research variables, this method have been chosen as it is distinguished from other factorial analysis methods because it draws the maximum variation of as explained by Safwat Farag (1985, p99)

Kaiser criterion proposed by Guttman used to determine accepted factors, and this criterion accept factors that have Eigen values more than 1.00 (R: 129)

Since the number of factors expected is directly linked to number of tests used in accordance with the following equation:

$$
r=\frac{1}{2}[(2 n+1)-\sqrt{8 n+1}]
$$

Where $\quad r=$ number of factors $\mathrm{n}=$ number of tests.

\section{Orthogonal Rotation}

Orthogonal Rotation is rotation of Axes lead to form of simpler and more systematic extracted factors and this facilitates interpretation of factors according to the appropriate reference framework to get closer solutions for building Simple Factorial Structure, then rotate the factors perpendicular with Varimax Rotation (Safwat Farag, 1985: p124).

It is clear from the reference framework available to researchers that orthogonal rotation is the most commonly used type of rotation in physical education research and studies.

Safwat Farag (1985, p261) explains that orthogonal rotation retain an angle of $\left(90^{\circ}\right)$ between the axes and as the angle cosine equal to zero, this means that the correlation between any perpendicular factors equal to zero, and this means that extracted factors by this method of rotation are independent and not overlapping factors. (Ahmed Sayed Ahmed, 2005: pp295300)

Tables (4), (8), (12) factor matrixes after orthogonal rotation using Varimax method for research sample, and loading values on the five extracted factors .

Factors interpretation and determining of morphological measurements:

Extracted factors through orthogonal rotation were interpreted using the following conditions:

- Following Thurston instructions referred to by Safwat farag $(1985,278)$ which represented in economy in factorial description, highlight unique aspects and different factor loading with concentration on factors that have meaning.(Safwat farag,1985: p278)

- Safawat Farag (1985, p365) quoting from Guilford indicates that we can - through the factor rotated matrix - factors characteristics and define its identity, so the factor is accepted only if there are three factors - at least 0loaded in it provided that loading of each of them is not less $( \pm 0.3)$

In light of the previous terms to accept factor, there were three (3) accepted factors, table (4) demonstrates these factors according to their order in the factor matrix. 
- Table (3) demonstrates that abdomen depth loaded on first factor with percentage of $(37.27 \%)$ from total research variables, while skinfolds thickness for iliac prominence loaded on second factor with percentage of $(25.01 \%)$ of total research variables, and overall height loaded in third factor with percentage of $(18.22 \%)$ from total variables.

- It is shown by this study that morphological factors which have highest loading and will be used as indicators for selection process will be consisted of number of measurements representing the extracted factors, i.e. one test or each factor, with the highest loading as shown in table (4)

Accordingly, the units selected in each morphological measurement extracted in this study are as its loading on other factors is not essential.

\section{Factors interpretation and determining of physiological measurements:}

Extracted factors through orthogonal rotation were interpreted using the following conditions:

- Following Thurston instructions referred to by Safwat farag $(1985,278)$ which represented in economy in factorial description, highlight unique aspects and different factor loading with concentration on factors that have meaning.(Safwat farag,1985: p278)

In light of the previous terms to accept factor, there were one (1) accepted factor, table (8) demonstrates these factors according to their order in the factor matrix.

- Table (7) demonstrates that emotional stability loaded on first factor with percentage of $(77.93 \%)$ from total research variables. It is shown by this study that physiological factors which have highest loading and will be used as indicators for selection process will be consisted of number of measurements representing the extracted factors, i.e. one test or each factor, with the highest loading as shown in table (8)

Accordingly, the units selected in each morphological measurement extracted in this study are as its loading on other factors is not essential.

\section{Factors interpretation and determining of physical measurements:}

Extracted factors through orthogonal rotation were interpreted using the following conditions:

- Following Thurston instructions referred to by Safwat farag $(1985,278)$ which represented in economy in factorial description, highlight unique aspects and different factor loading with concentration on factors that have meaning.(Safwat farag,1985: p278)

- Safawat Farag (1985, p365) quoting from Guilford indicates that we can - through the factor rotated matrix - factors characteristics and define its identity, so the factor is accepted only if there are three factors - at least 0loaded in it provided that loading of each of them is not less $( \pm 0.3)$

In light of the previous terms to accept factor, there were one (1) accepted factors, table (12) demonstrates these factors according to their order in the factor matrix.

- Table (11) demonstrates that speed endurance loaded on first factor with percentage of $(60.1 \%)$ from total research variables. It is shown by this study that morphological factors which have highest loading and will be used as indicators for selection process will be consisted of number of measurements representing the extracted factors, i.e. one test or each factor, with the highest loading as shown in table (12)

From the previous interpretation for the five factors and based on morphological, physiological and physical measurements, and and extracting highest loaded variable from each factor; it is possible to determine five factors as it is demonstrated in table (4), (8), and (12) which includes: (Speed endurance, Abdomen depth, Skinfolds thickness for iliac prominence, Overall height and Emotional stability)

\section{Conclusions}


As per factor analysis of this study data and within study objective and procedures, and statistical results, researchers could conclude the following:

Accepting five basic factors in Biological and physical measurements for Handball goalkeepers, namely:

\section{First: Biological measurements:}

- Determined morphological measurements were: (Abdomen depth, Skin folds thickness for iliac prominence, Overall height)

- Determined physiological measurements were: emotional stability

\section{Second: the physical variables:}

- Determined physical measurements were: speed endurance.

\section{Recommendations}

\section{In light of conclusions reached in this study the following is recommended:}

There is necessity to focus on Biological( morphological, physiological ) and physical criteria extracted in this study (Speed endurance, Abdomen depth, Skin folds thickness for iliac prominence, Overall height and Emotional stability) which could represent scientific base of:

1. Using extracted measurements in selection of handball goalkeepers

2. Giving attention to Biological (morphological, physiological) and physical aspects as an important indicator for players' general physical condition.

3. Selection process based on sound scientific principles.

4. Choosing appropriate anticipated handball goalkeepers .

5. Organizing and directing training programs for handball goalkeepers and upgrading handball sport at national level.

6. Continuing achievements and winning world championships.
7. Further scientific studies on Biological (morphological, physiological) and physical criteria for handball international teams' goalkeeper and comparing it with handball national teams' handball goalkeepers and then take advantage of them scientifically and practically.

\section{References}

1. Abul Ela Ahmed Abd Elfattah (1988): sports biology - 3rd ed. - Dar Elfikr El Arabi, Cairo, (in Arabic).

2. Abul Ela Ahmed Abd Elfattah, Mohamed Sobhy Hassanein (1997) sport physiology and morphology, and measurement and evaluation methods - 1st ed.- Dar Elfikr El Arabi , Cairo. (in Arabic).

3. Ahmed Mohamed Said Ahmed (2005): Factor analysis of sports physiological tests, theories and applications, Faculty of Physical Education for Men, Abu Qir, Alexandria . (in Arabic)

4. Ali Fahmy El Bek (1996): comparative study for some anthropometric measurements at of high level swimmers and Saudi long distance swimming, scientific research - Helwan magazine - Volume III - No 2, August. (in Arabic).

5. Baumgartner, T.A. \& Jackson, AS (1995): Measurement for Evaluation 5th., Ed., Brown \& Benchmark Iowa.

6. Blair, V.A.,(1999): Hand Function. In Functional Human Movement Edited by Durward BR, Baer, GD, \& Rowe, PJ, Butterworth Heinemann, Oxford, UK.

7. Davis, B., et al (1995): Physical Education and Study of Sorts, 2nd ed., Mosby, New york, Philadelphia, USA.

8. Foss, M.L. \& Keteyian, S. J., (1998):- Fox's Physiological Basis for Exercise and Sport, 6th., Ed., MGrow-Hill Publishing Co., New York.

9. Hazaa Bin Mohammed Al-Hazaa (2010): Exercise Physiology -theoretical basis and laboratory measures of physiological measurements,- Part I, scientific publishing and 
printing presses,- King Saud University. (in Arabic).

10. Kamal Derwish, Mohammed Ahmed Abdo Khalil, Imad Eddin Abbas - Sami Mohammed Ali(1998): handball goalkeeper, Ketab publication Center, Cairo. (in Arabic).

11. Miller PD, (1995): Fitness Programming and Physical Disability, Human Kinetics, Champaign.

12. Mohamed Lotfi Taha(2002): Psychological foundations for athletes selection, Cairo. (in Arabic).

13. Mohamed Sobhy Hassanin (1995): Measurement and Evaluation in Physical Education and Sports, Part II, Third Edition, Dar Elfikr El Arabi, Cairo. (in Arabic).

14. Mohamed Sobhy Hassanin(2000): Measurement and Evaluation in Physical Education and Sports, Dar Elfikr El Arabi, Cairo. (in Arabic).

15. Mohamed Sobhy Hassanin(2003): Measurement and Evaluation in Physical Education and Sports , Part II, 5th ed., Dar Elfikr El Arabi, Cairo. (in Arabic).

Mohamed Sobhy Hassanin (2004): Measurement and Evaluation in Physical Education and Sports
- Part I - 6th ed. , Dar Elfikr El Arabi, Cairo. (in Arabic).

Mohammed Samir Saad Eldin (1997): physiology and physical effort, 2nd edition, Monshaat El Maaref, Alexandria. (in Arabic).

Munir Gerges Ibrahim (2004): Handball for all, Dar Elfikr El Arabi, Cairo. (in Arabic).

Rasha Abdullah Antar Emira (2009): some anthropometric and physical characteristics of handball juniors in different play positions, Master thesis, Faculty of Physical Education for Girls, Alexandria University. (in Arabic).

Safwat Muhammad Faraj (1985): factor analysis in behavioral sciences, 2nd ed., the AngloEgyptian Library, Cairo . (in Arabic).

Sherwood, L., (2001): Human Physiology, from Cells to System, 4th., Ed., Brooks-Cole Publishing Co., New York, 2001.

Ted a.Bau mgartner \& Andrews.Jason(1999): Measurement For Evaluation and Exercise sience WCB BROWN \& Benchnark.

Zakia Ahmed Fathy,- Mahmoud Abdel-Hafiz alNajjar(2001): $\quad$ Sport Physiology (applications),1st ed. ,Al Ghad Press, Alexandria. (in Arabic). 